\title{
Infrared Optical Response of Metallic Graphene Nanoribbons
}

\author{
Zigang Duan, ${ }^{1}$ Wenhu Liao, ${ }^{2}$ and Guanghui $\mathrm{Zhou}^{2}$ \\ ${ }^{1}$ Key Laboratory of Optoelectronic Devices and Systems of Ministry of Education and Guangdong Province, Shenzhen University, \\ Shenzhen 518060, China \\ ${ }^{2}$ Department of Physics and Key Laboratory for Low-Dimensional Structures and Quantum Manipulation (Ministry of Education), \\ Hunan Normal University, Changsha 410081, China
}

Correspondence should be addressed to Guanghui Zhou, ghzhou@hunnu.edu.cn

Received 22 December 2009; Revised 25 April 2010; Accepted 25 April 2010

Academic Editor: Yuri Galperin

Copyright (c) 2010 Zigang Duan et al. This is an open access article distributed under the Creative Commons Attribution License, which permits unrestricted use, distribution, and reproduction in any medium, provided the original work is properly cited.

\begin{abstract}
We investigate theoretically the infrared optical response characteristics of metallic armchair/zigzag-edge graphene nanoribbons (A/ZGNRs) to an external longitudinally polarized electromagnetic field at low temperatures. Within the framework of linear response theory at the perturbation regime, we examine the optical infrared absorption threshold energy, absorption power, dielectric function, and electron energy loss spectra near the neutrality points of the systems. It is demonstrated that, by some numerical examples, the photon-assisted direct interband absorptions for AGNR exist with different selection rules from those for ZGNR and single-walled carbon nanotube at infrared regime. This infrared optical property dependence of GNRs on field frequency may be used to design graphene-based nanoscale optoelectronic devices for the detection of infrared electromagnetic irradiations.
\end{abstract}

\section{Introduction}

Intriguing experimental [1] and theoretical [2-11] attentions have been paid to graphene-based electronics, especially the unique electronic [3-11] and transport properties [12-19] of GNRs due to its potential applications in nanodevices. The optical spectra [20] of semiconducting GNRs and the excitonic effects $[21,22]$ in the optical properties of AGNRs with many-electron effects included have been investigated by density functional theory and the firstprinciples calculations, respectively. Liu et al. [23] have demonstrated that magnetic field can enhance and tune the optical response of GNRs in the terahertz to far-infrared regime. The effects of intrinsic spin-orbit and Coulomb interactions on low-energy properties of AGNRs have been studied [24]. The optical absorption selection rule [25] for ZGNR has been proposed to be qualitatively different from that for armchair carbon nonotubes (CNTs) [26, 27]. The optical properties of AGNRs [28] irradiated under an longitudinal polarized electromagnetic field have also been analyzed. Zhang et al. [29] have investigated the magnetooptical properties of graphene quantum dots. Furthermore, the optical response of the ZGNRs with the spin interaction included has been found to be dominated by magnetic edge-state-derived excitons other than the affections of the edges and confinement on the optical transition energies. $[30,31]$ However, to the best of our acknowledge, the optical properties for driven GNRs [20-25, 28-31] in the important frequency band of infrared have seldom been involved.

A N-AGNR is either semiconducting or metallic depending on the number of dimer lines $N$ along the edge. However, an $N$-ZGNR is always metallic due to its edge states. In this paper, we present a theoretical investigation on the infrared properties in the vicinity of neutrality points for the two types of metallic GNRs under the irradiation of an external longitudinal polarized low-frequency field at low temperatures. The dependence of the optical absorption threshold values, absorption power, dielectric function, and electron energy loss spectrum (EELS) on the irradiation energy are demonstrated in the framework of linear response theory under the dipole approximation [25, 28, 29]. Some new interband absorptions are predicted with the exception of the quantitative description of threshold energy on the width of GNRs, and the results are discussed and compared with those in the previous works [20-31]. 
The rest of the paper is organized as follows. The analytical expressions of the band spectra close to the neutrality points for A/ZGNRs, the optical absorption power, dielectric function, and electron energy loss spectra are calculated in Section 2. Some numerical examples and discussions for the results are demonstrated in Section 3. Finally, Section 4 concludes the paper.

\section{Model and Formulism}

The tight-binding $\pi$ electron dispersion relation for an ideal infinite graphene sheet can be solved exactly as $[3,4,10,11]$

$$
\begin{aligned}
& \epsilon_{k_{x}, k_{y}} \\
& = \pm \gamma\left[3+2 \cos \left(k_{y} a\right) \pm 4\left|\cos \left(\frac{k_{y} a}{2}\right) \cos \left(\frac{\sqrt{3} k_{x} a}{2}\right)\right|\right]^{1 / 2},
\end{aligned}
$$

where the hopping integral $\gamma=2.75 \mathrm{eV}$, the minimal translation distance of honeycomb lattice is $a=2.46 \AA$, and the variation range of $k_{x}$ and $k_{y}$ should be specified for particular GNRs.

Employing the hard-wall boundary conditions to longitudinal wave function for a $N$-AGNR, that is, $\phi_{A / B}(0)=$ $\phi_{A / B}(N+1)=0$, one gets the discretized wave vector $k_{y}=m \pi$ / $[(N+1) a]$, and further obtains the rationalized band spectrum in terms of continuous $k_{x}$ (longitudinal) and discrete $m$ (transversal) quantum numbers near the point of neutrality $[10,11]$

$$
\begin{aligned}
& \epsilon_{n, k_{x}} \\
& = \pm \gamma\left[3+2 \cos \frac{\left(j^{*}+n\right) \pi}{N+1}-4 \cos \frac{\left(j^{*}+n\right) \pi}{2(N+1)} \cos \frac{\sqrt{3} k_{x} a}{2}\right]^{1 / 2},
\end{aligned}
$$

where \pm represents, respectively, the conduction bands (CB) and valence bands $(\mathrm{VB}), j^{*}=2(N+1) / 3$ is the lowest/highest $\mathrm{CB} / \mathrm{VB}$ mode with $n=0,1,2, \ldots, N-j^{*}$, and the longitudinal wave vector is confined within the first Brillouin zone $0 \leq$ $\left|\sqrt{3} k_{x} a\right| \leq \pi$.

Distinct from AGNR, the discrete values of $k_{y}$ for an $N$ ZGNR are $k_{x}$-dependent, $[10,11]$ which is determined by

$$
\frac{\sin \left(\kappa_{n} N\right)}{\sin \left[\kappa_{n}(N+1 / 2)\right]}= \pm 2 \cos \left(\frac{k_{x} a}{2}\right),
$$

where $\kappa_{n}=\sqrt{3} k_{y} a$. Therefore, one obtains $\epsilon_{n, k_{x}}= \pm \gamma \sin \left(\kappa_{n} /\right.$ $2) / \sin \left[\kappa_{n}(N+1 / 2)\right]$ by substituting (3) into (1) and the real solutions $\kappa_{n}=(n+1 / 2) \pi / N$ of the extrema for the conduction bands by equation $\sin \left(\kappa_{n} N\right) / \sin \left[\kappa_{n}(N+1)\right]=N /(N+1)$, where $n=0,1,2, \ldots, n \ll N$. Therefore, the band spectrum for a $N-Z G N R$ can be induced as $[10,11]$

$$
\begin{aligned}
& \epsilon_{n, k_{x}} \\
& = \pm \gamma\left[\frac{3}{4}\left(k_{x} a-\frac{2 \pi}{3}\right)^{2}+\frac{(n+1 / 2)^{2} \pi^{2}}{4 N^{2}}\left(1+\frac{\sqrt{3} \pi}{3}-\frac{\sqrt{3} k_{x} a}{2}\right)\right]^{1 / 2},
\end{aligned}
$$

with the exception of the edge states and the continuous variable $k_{x}$ is restricted to the range of $0 \leq\left|k_{x} a\right| \leq \pi$. However, when $k_{x}$ falls into the interval $2 \pi / 3<\left|k_{x} a\right| \leq \pi$, the imaginary solution of (3) reads

$$
\epsilon_{0, k_{x}}= \pm \gamma \frac{\sinh \left[-\ln \left[2 \cos \left(k_{x} a / 2\right)\right]\right]}{\sinh \left[-(2 N+1) \ln \left[2 \cos \left(k_{x} a / 2\right)\right]\right]},
$$

which gives the edge states (make ZGNRs always metallic) localized on the edge and decay exponentially into the center. The \pm in (4) and (5) represents the CB and VB, respectively.

Using the perturbation theorem in the dipole-transition approximation, the optical absorption power for perfect GNRs irradiated under a longitudinally polarized weak electromagnetic field can be expressed as

$$
\begin{aligned}
P(\omega)= & \frac{4 \pi e^{2} E_{0}^{2}}{m_{e}^{2} \omega} \sum_{n_{1}, n_{2}, k_{x}}\left[f\left(\epsilon_{n_{1}, k_{x}}\right)-f\left(\epsilon_{n_{2}, k_{x}}\right)\right] \\
& \times\left|\left\langle n_{1}, k_{x}\left|\hat{p}_{x}\right| n_{2}, k_{x}\right\rangle\right|^{2} \delta\left(\epsilon_{n_{2}, k_{x}}-\epsilon_{n_{1}, k_{x}}-\hbar \omega\right),
\end{aligned}
$$

where $E_{0}$ and $\omega$ are the intensity and frequency of the irradiation field, respectively, $m_{e}$ is the free-electron mass, $f\left(\epsilon_{n, k_{x}}\right)$ the Fermi-Dirac distribution function, and $n_{1 / 2}$ the subband indices for the $\mathrm{VB} / \mathrm{CB}$, while $\hat{p}_{x}$ is the $x$-component of the momentum operator. Furthermore, the imaginary part of the complex dielectric function $\xi_{2}(\omega)$ can be obtained as

$$
\begin{aligned}
\xi_{2}(\omega)= & \frac{8 \pi^{2} e^{2}}{m_{e}^{2} \omega^{2}} \sum_{n_{1}, n_{2}, k_{x}}\left[f\left(\epsilon_{n_{1}, k_{x}}\right)-f\left(\epsilon_{n_{2}, k_{x}}\right)\right] \\
& \times\left|\left\langle n_{1}, k_{x}\left|\hat{p}_{x}\right| n_{2}, k_{x}\right\rangle\right|^{2} \delta\left(\epsilon_{n_{2}, k_{x}}-\epsilon_{n_{1}, k_{x}}-\hbar \omega\right),
\end{aligned}
$$

and one gets the real part of the dielectric function $\xi_{1}(\omega)$ by Kramers-Kronig transformation [26-28]

$$
\xi_{1}(\omega)=1+\frac{4}{\pi} \wp \int_{0}^{\infty} \frac{\omega^{\prime} \xi_{2}\left(\omega^{\prime}\right)}{\omega^{\prime 2}-\omega^{2}} d \omega^{\prime},
$$

where $\wp$ denotes the integral principal value. Therefore, at the long-wavelength limit [26-28], one can obtain EELS by $-\Im m\left[\xi(\omega)^{-1}\right]$ while $\Im m$ represents the imaginary part, the refractive index, and reflectivity from the complex dielectric function $\xi$ for the systems.

\section{Results and Discussions}

In the following, we present the numerical examples of the calculated $P, \xi_{2}$, and EELS for two types of GNRs under the irradiation of a longitudinal polarized electromagnetic field. In the calculation, the Dirac delta function in (6) and (7) is simulated [26-28] as $\delta(x)=e^{-x^{2} \Gamma^{-2}} /\left(\Gamma \pi^{1 / 2}\right)$ with the Gaussian broadening factor $\Gamma=0.014 \mathrm{eV}$. The coupling between the $s p^{2}$ states and the $p_{z}$ state is neglected since we are only interested in the optical response of the GNRs near the zero-energy points, that is, at the low energy regime [29]. Under the irradiation of a weak electromagnetic 


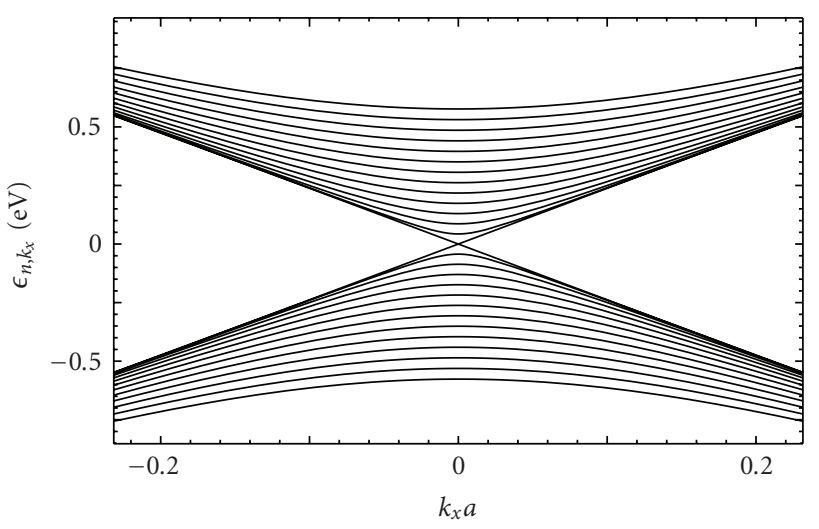

(a)

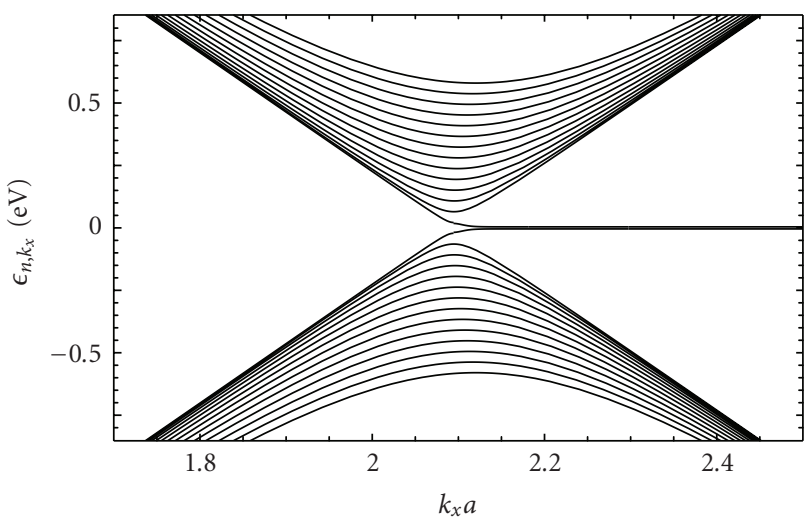

(b)

FIGURE 1: Electron band structures for perfect (a) 173-AGNR and (b) 100-ZGNR. The lattice constant along the nanoribbons is $a=$ $2.46 \AA$.

field, the dipole transition matrix elements $\left\langle n_{1}, k_{x}\left|\hat{p}_{x}\right| n_{2}, k_{x}\right\rangle$ within the tight-binding single-electron picture $[25,28]$ are chosen as 0.206. Since the wavelength (several hundreds of nanometer) of the weak infrared field is much larger than the transversal size (about 42.5 nanometer) of the 173-AGNR and 100-ZGNR, one can ignore the local-field correction in the present systems. [21, 22, 26, 27] Furthermore, the excitonic effects can also be ignored since the electronelectron interaction has not been included in this work [21, 22, 26, 27].

In Figure 1, we present the electronic structures $\epsilon_{n, k_{x}}$ versus the longitudinal wave vector $k_{x}$ near the neutrality points of 173-AGNR and 100-ZGNR in panel (a) and (b), respectively. As is seen the 173-AGNR case from Figure 1(a), the $\mathrm{CB}$, and the $\mathrm{VB}$ are mirror symmetric with respect to the Fermi level $E_{F}=0$, and the $\mathrm{CB}$ subbands at $k_{x}$ $=0$ correspond (from bottom to top in sequential order) to $n=0,1,2, \ldots, 13$. Moreover, Figure $1(\mathrm{~b})$ the $100-$ ZGNR case presents an armchair-CNT-like [25] electron band structure other than the lowest/highest conduction/valence band, which converts from an almost linear decrease/increase for $k_{x} a \leq 2.09$ to an exponential-like (governed by (5)) curve up to the edge of the first Brillouin zone, while the sequence for the other subbands of the $\mathrm{CB}$ is $n=1,2, \ldots, 13$ from

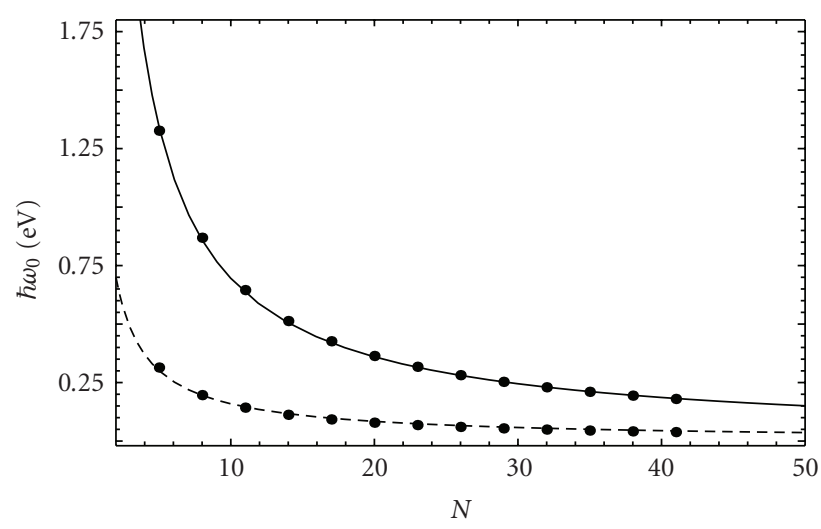

FIGURE 2: Threshold energy for GNRs as a function of their width. The solid line fits for AGNRs and the dashed line for ZGNRs.

bottom to top. When the electromagnetic field is polarized longitudinally, the allowed optical absorptions for CNTs $[26,27]$ are restricted to vertical excitations (i.e., $n$ and $k_{x}$ remain unchanged) between the $\mathrm{VB}$ and the $\mathrm{CB}$, while those for ZGNRs [25] are from $V_{0}\left(V_{\text {odd }}\right)$ to $C_{\text {odd }}\left(C_{0}\right)$ in the low energy range, where $V_{0}$ and $C_{0}$ denote the highest valence and the lowest conduction subband, respectively, while the oddth and eventh conduction and valence subbands for GNRs are denoted as $C / V_{\text {odd }}$ and $C / V_{\text {even }}$. As follows, different optical absorptions will be demonstrated from the calculated $P(\omega)$ and $\xi_{2}(\omega)$ at vanishing $\partial \epsilon_{n, k_{x}} / \partial k_{x}$ for longitudinally irradiated A/ZGNRs.

The threshold energy $\hbar \omega_{0}$ (the optical transition energy between the highest valence subband and the second conduction subband or that from the second highest valence subband to the lowest conduction one for AGNR, while it is the optical transition from/to the edge states for ZGNR) as a function of the GNRs width is shown in Figure 2. As expected, the wider the ribbons, the lower the threshold energy (decreasing from visible to infrared). It is observed that the discretized points can be fitted by $6.20 \times N^{-0.95}$ and $1.32 \times N^{-0.92}$ for AGNRs (see the solid line in Figure 2) and ZGNRs (the dashed line in Figure 2), respectively, therefore, the threshold energy for AGNRs is more sensitive to width than that for ZGNRs since the electronic properties of AGNRs are more sensitive to their geometries [20,21].

The optical absorption power $P$ (in arbitrary units) near the neutrality points of 173-AGNR and 100-ZGNR as a function of the irradiation energy is demonstrated in Figure 3. In the presence of an external irradiation field, a peak in $P(\omega)$ indicates a direct absorption photon with energy $\hbar \omega$ followed by a transition from the VB to the CB. As illustrated in Figure 3(a) for the 173-AGNR case, the absorption peaks at $0.041,0.129,0.217,0.305,0.393,0.484$, and $0.575 \mathrm{eV}$ can be identified to the transitions $V_{0}\left(V_{i}\right) \rightarrow$ $C_{i}\left(C_{0}\right)$, as denoted by $p_{0 i}$ with $i=1,3, \ldots, 13$ in Figure $3(\mathrm{a})$, while those at $0.085,0.173,0.261,0.349,0.44$, and $0.531 \mathrm{eV}$ result from the excitations $V_{0}\left(V_{j}\right) \rightarrow C_{j}\left(C_{0}\right)$ as denoted by $p_{0 j}$ with $j=2,4, \ldots, 12$ in Figure $3(\mathrm{a})$, respectively. It is worthwhile to note that some vertical transitions (with unchanged $k_{x}$ and subband indices) have been shown. 


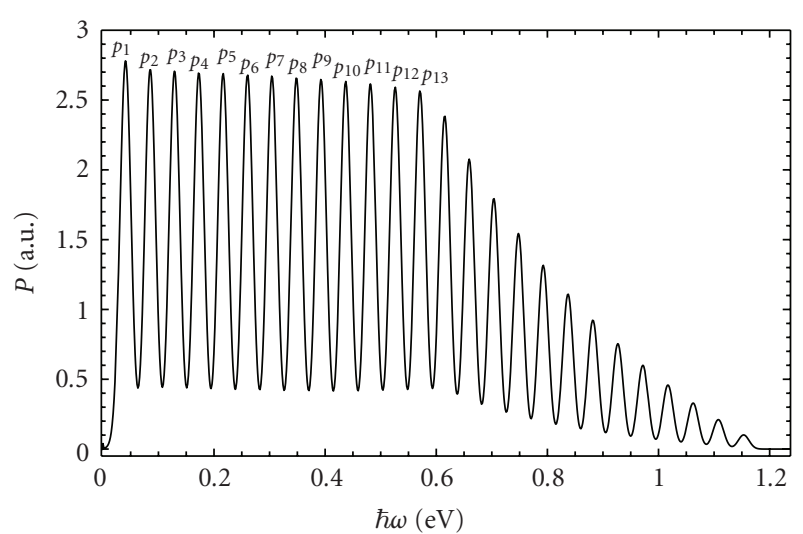

(a)

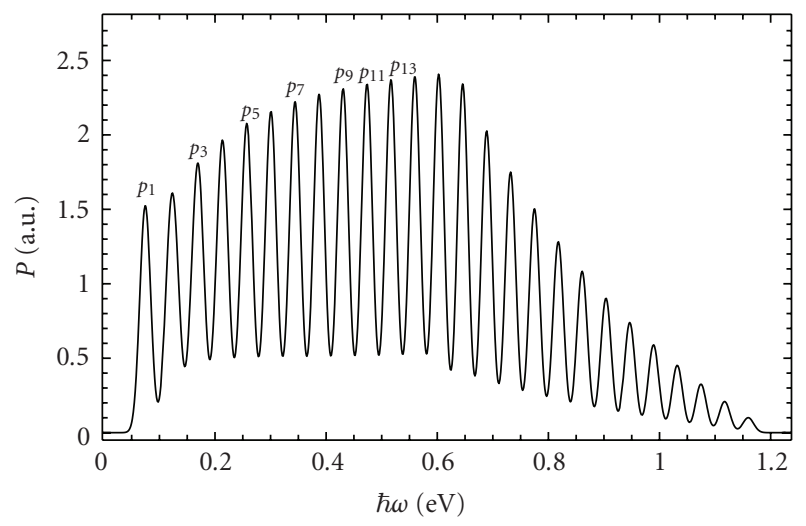

(b)

FIGURE 3: Optical absorption power for (a) 173-AGNR and (b) 100ZGNR as a function of the irradiation energy.

For example, the peaks at $0.616,0.792,0.974$, and $1.152 \mathrm{eV}$ should rely on the excitations from $V_{7} \rightarrow C_{7}, V_{9} \rightarrow C_{9}$, $V_{11} \rightarrow C_{11}$, and $V_{13} \rightarrow C_{13}$, while the absorptions at 0.704 , 0.88 , and $1.062 \mathrm{eV}$ may be from $V_{8} \rightarrow C_{8}, V_{10} \rightarrow C_{10}$, and $V_{12} \rightarrow C_{12}$, respectively. However, one can owe the peaks at $0.66,0.748,0.836,0.924,1.015$, and $1.108 \mathrm{eV}$ to the transitions from $V_{\text {odd }}\left(V_{\text {even }}\right)$ to $C_{\text {even }}\left(C_{\text {odd }}\right)$ similarly as [28]. Furthermore, Figure 3(b) exhibits the 100-ZGNR case. One can identify those absorption peaks at $0.074,0.168,0.25$, $0.347,0.429,0.486$, and $0.512 \mathrm{eV}$ to the transitions between the decaying modes (edge states) and the odd $t h$ subbands, as denoted by $p_{0 i}$ with $i=1,3, \ldots, 13$ in Figure $3(\mathrm{~b})$, respectively. The resonance structures at $0.072,0.215,0.303,0.388,0.476$, $0.561,0.646,0.732,0.817,0.902,0.99$, and $1.075 \mathrm{eV}$ may be attributed to the vertical transitions between the VB and the CB. It should be pointed out that the current results are different from the absorption coefficient of the monolayer graphene [32] in the optical range of frequencies due to the quantum confinement and different optical transitions. It is noted that the optical absorptions for AGNR are stronger than those for ZGNR at the regime of $0<\hbar \omega<0.66 \mathrm{eV}$ and decrease slower in the higher energy range.

Furthermore, the imaginary part of the dielectric function $\xi_{2}$ (in arbitrary units) as a function of photon energy

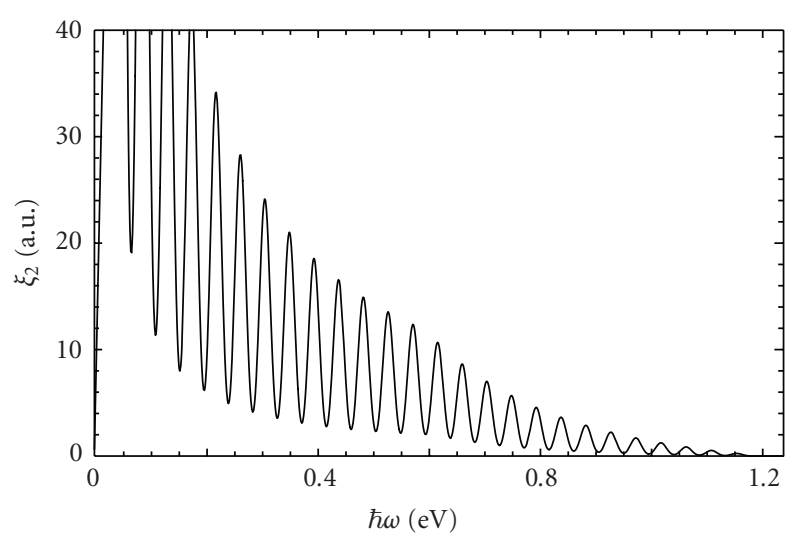

(a)

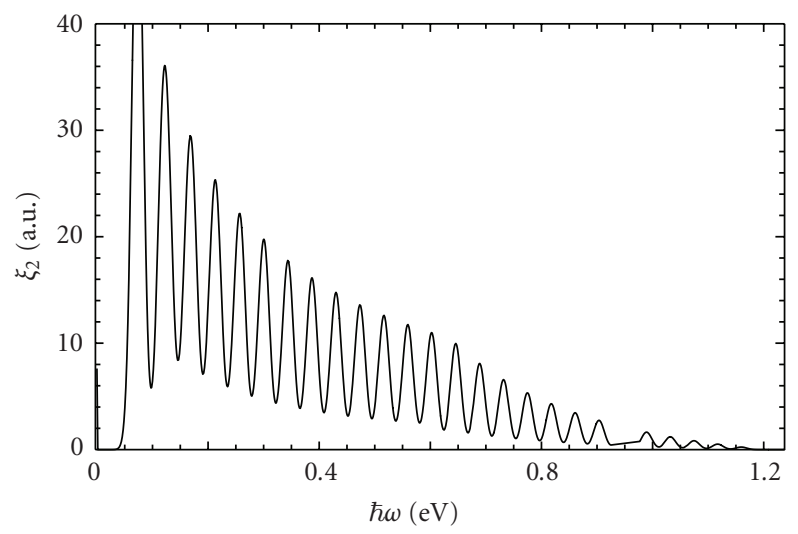

(b)

FIGURE 4: Imaginary part of the dielectric function for (a) 173AGNR and (b) 100-ZGNR as a function of photon energy.

is illustrated in Figure 4. As shown in Figure 4(a) for the 173-ANGR case, one notes a series of resonance structures, corresponding to the absorption peaks in Figure 3(a), which are much higher in the low energy range since $\xi_{2} \propto P / \omega$. In correspondence to the optical transitions in Figure 3(b), the 100-ZGNR case (see Figure 4(b)) presents a set of relative lower resonance peaks, especially at the low energy regime. It seems that AGNR is more sensitive to the low frequency infrared than ZGNR, which is consistent with $[23,28]$. One notices that the imaginary dielectric function $\xi_{2}$ for both 173-AGNR and 100-ZGNR demonstrates several zero points (corresponding to plasma frequencies of the systems) at the higher energy regime.

The EELS of the two systems as a function of the irradiation field energy is demonstrated finally in Figure 5. Since the system EELS is combined with the complex dielectric function $\xi$ simply by $-\Im m\left[\xi^{-1}\right]$ (see [26-28]), Figure 5(a) (the 173-AGNR case) shows a set of singular peaks at $0.55,0.688,0.77,0.825,0.853,0.88,0.935,0.963,0.99$, $1.018,1.045$, and $1.155 \mathrm{eV}$ corresponding to the plasmon frequencies (zero points of $\xi_{1}$, not shown here) while the 100-ZGNR case (see Figure 5(b)) presents some sharp peaks at $0.765,0.798,0.847,0.963,0.99$, and $1.073 \mathrm{eV}$ with much lower ones at $0.935,1.018$, and $1.155 \mathrm{eV}$ than the 173-AGNR 


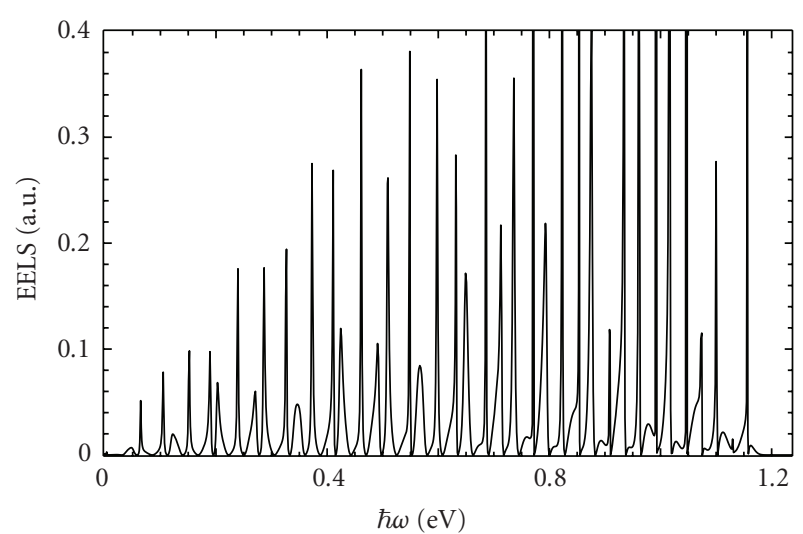

(a)

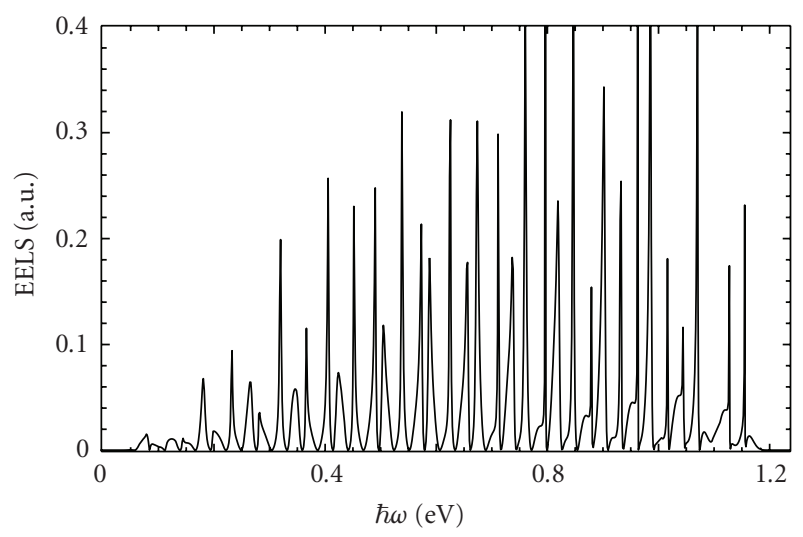

(b)

FIGURE 5: EELS for (a) 173-AGNR and (b) 100-ZGNR as a function of the irradiation energy.

case. It seems that there are more plasmon modes for AGNR than for ZGNR in the infrared range, while the fine details $[26,27]$ of the system EELS may be smoothed out by a larger broadening parameter.

\section{Conclusion}

In summary, using the linear response theory, we have investigated theoretically the optical properties of semiinfinite clean A/ZGNR under the irradiation of an external longitudinal polarized low-frequency electromagnetic field at low temperatures. Under the dipole-transition approximation, it is shown that the optical absorption power, dielectric function and electron energy loss spectrum of the systems are sensitive to the infrared irradiation depending on the chirality and the width of GNRs. Some new photonassisted direct interband transitions are proposed. The predicted optical properties are expected to be observed by scanning tunneling microscopy optical spectroscopy $[33,34]$ and reflection contrast spectroscopy [35] experiments and used to design the graphene-based nanoscale optoelectronic devices [36-38].

\section{Acknowledgments}

This work was supported by the National Natural Science Foundation of China (Grant no. 10974052), Specialized Research Fund for the Doctoral Program of Higher Education of China (Grant no. 20060542002).

\section{References}

[1] Y.-M. Lin, V. Perebeinos, Z. Chen, and P. Avouris, "Electrical observation of subband formation in graphene nanoribbons," Physical Review B, vol. 78, no. 16, Article ID 161409, 2008.

[2] Y.-W. Son, M. L. Cohen, and S. G. Louie, "Half-metallic graphene nanoribbons," Nature, vol. 444, no. 7117, pp. 347349, 2006.

[3] L. Brey and H. A. Fertig, "Electronic states of graphene nanoribbons studied with the Dirac equation," Physical Review $B$, vol. 73, no. 23, Article ID 235411, 2006.

[4] L. Yang, C.-H. Park, Y.-W. Son, M. L. Cohen, and S. G. Louie, "Quasiparticle energies and band gaps in graphene nanoribbons," Physical Review Letters, vol. 99, no. 18, Article ID 186801, 2007.

[5] Y.-W. Son, M. L. Cohen, and S. G. Louie, "Energy gaps in graphene nanoribbons," Physical Review Letters, vol. 97, no. 21, Article ID 216803, 2006.

[6] D. Gunlycke and C. T. White, "Tight-binding energy dispersions of armchair-edge graphene nanostrips," Physical Review B, vol. 77, no. 11, Article ID 115116, 2008.

[7] M. Y. Han, B. Özyilmaz, Y. Zhang, and P. Kim, "Energy bandgap engineering of graphene nanoribbons," Physical Review Letters, vol. 98, no. 20, Article ID 206805, 2007.

[8] K. Nakada, M. Fujita, G. Dresselhaus, and M. S. Dresselhaus, "Edge state in graphene ribbons: nanometer size effect and edge shape dependence," Physical Review B, vol. 54, no. 24, pp. 17954-17961, 1996.

[9] S. Okada, "Energetics of nanoscale graphene ribbons: edge geometries and electronic structures," Physical Review B , vol. 77, no. 4, Article ID 041408, 2008.

[10] L. Malysheva and A. Onipko, "Spectrum of $\pi$ Electrons in graphene as a macromolecule," Physical Review Letters, vol. 100, no. 18, Article ID 186806, 2008.

[11] L. I. Malysheva and A. I. Onipko, "Quantum conductance of achiral graphene ribbons and carbon tubes," Journal of Experimental and Theoretical Physics, vol. 108, no. 1, pp. 126131, 2009.

[12] Y. P. Chen, Y. E. Xie, and X. H. Yan, "Electron transport of Lshaped graphene nanoribbons," Journal of Applied Physics, vol. 103, no. 6, Article ID 063711, 2008.

[13] H. Xu, T. Heinzel, M. Evaldsson, and I. V. Zozoulenko, "Magnetic barriers in graphene nanoribbons: theoretical study of transport properties," Physical Review B, vol. 77, no. 24, Article ID 245401, 2008.

[14] F. Zhai and K. Chang, "Theory of huge tunneling magnetoresistance in graphene," Physical Review B, vol. 77, no. 11, Article ID 113409, 2008.

[15] L. Brey and H. A. Fertig, "Elementary electronic excitations in graphene nanoribbons," Physical Review B, vol. 75, no. 12, Article ID 125434, 2007.

[16] Q. Liang, Y. Yu, Q. Wang, and J. Dong, "Controllable $0-\pi$ transition in a superconducting graphene-nanoribbon junction," Physical Review Letters, vol. 101, no. 18, Article ID 187002, 2008. 
[17] M. Zarea, C. Büsser, and N. Sandler, "Unscreened coulomb interactions and the quantum spin hall phase in neutral zigzag graphene ribbons," Physical Review Letters, vol. 101, no. 19, Article ID 196804, 2008.

[18] Z. Li, H. Qian, J. Wu, B.-L. Gu, and W. Duan, "Role of symmetry in the transport properties of graphene nanoribbons under bias," Physical Review Letters, vol. 100, no. 20, Article ID 206802, 2008.

[19] E.-J. Kan, Z. Li, J. Yang, and J. G. Hou, "Will zigzag graphene nanoribbon turn to half metal under electric field?" Applied Physics Letters, vol. 91, no. 24, Article ID 243116, 2007.

[20] V. Barone, O. Hod, and G. E. Scuseria, "Electronic structure and stability of semiconducting graphene nanoribbons," Nano Letters, vol. 6, no. 12, pp. 2748-2754, 2006.

[21] L. Yang, M. L. Cohen, and S. G. Louie, "Excitonic effects in the optical spectra of graphene nanoribbons," Nano Letters, vol. 7, no. 10, pp. 3112-3115, 2007.

[22] D. Prezzi, D. Varsano, A. Ruini, A. Marini, and E. Molinari, "Optical properties of graphene nanoribbons: the role of many-body effects," Physical Review B, vol. 77, no. 4, Article ID 041404, 2008.

[23] J. Liu, A. R. Wright, C. Zhang, and Z. Ma, "Strong terahertz conductance of graphene nanoribbons under a magnetic field," Applied Physics Letters, vol. 93, no. 4, Article ID 041106, 2008.

[24] M. Zarea and N. Sandler, "Electron-electron and Spin-orbit interactions in armchair graphene ribbons," Physical Review Letters, vol. 99, no. 25, Article ID 256804, 2007.

[25] H. Hsu and L. E. Reichl, "Selection rule for the optical absorption of graphene nanoribbons," Physical Review B, vol. 76, no. 4, Article ID 045418, 2007.

[26] M. F. Lin and K.W.-K. Shung, "Plasmons and optical properties of carbon nanotubes," Physical Review B, vol. 50, no. 23, pp. 17744-17747, 1994.

[27] G. Y. Guo, K. C. Chu, D.-S. Wang, and C.-G. Duan, "Linear and nonlinear optical properties of carbon nanotubes from first-principles calculations," Physical Review B, vol. 69, no. 20, Article ID 205416, 2004.

[28] W. Liao, G. Zhou, and F. Xi, "Optical properties for armchairedge graphene nanoribbons," Journal of Applied Physics, vol. 104, no. 12, Article ID 126105, 2008.

[29] Z. Z. Zhang, K. Chang, and F. M. Peeters, "Tuning of energy levels and optical properties of graphene quantum dots," Physical Review B, vol. 77, no. 23, Article ID 235411, 2008.

[30] L. Yang, M. L. Cohen, and S. G. Louie, "Magnetic edge-state excitons in zigzag graphene nanoribbons," Physical Review Letters, vol. 101, no. 18, Article ID 186401, 2008.

[31] J. Jiang, W. Lu, and J. Bernholc, "Edge states and optical transition energies in carbon nanoribbons," Physical Review Letters, vol. 101, no. 24, Article ID 246803, 2008.

[32] D. S. L. Abergel and V. I. Fal'ko, "Optical and magneto-optical far-infrared properties of bilayer graphene," Physical Review B, vol. 75, no. 15, Article ID 155430, 2007.

[33] R. Arenal, O. Stéphan, M. Kociak, D. Taverna, A. Loiseau, and C. Colliex, "Electron energy loss spectroscopy measurement of the optical gaps on individual boron nitride single-walled and multiwalled nanotubes," Physical Review Letters, vol. 95, no. 12, Article ID 127601, 4 pages, 2005.

[34] Z. Fei, Y. Shi, L. Pu et al., "High-energy optical conductivity of graphene determined by reflection contrast spectroscopy," Physical Review B, vol. 78, no. 20, Article ID 201402, 2008.
[35] Z. Fei, Y. Shi, L. Pu et al., "High-energy optical conductivity of graphene determined by reflection contrast spectroscopy," Physical Review B, vol. 78, no. 20, Article ID 201402, 2008.

[36] T. Yamamoto, T. Noguchi, and K. Watanabe, "Edge-state signature in optical absorption of nanographenes: tightbinding method and time-dependent density functional theory calculations," Physical Review B, vol. 74, no. 12, Article ID 121409, 2006.

[37] S. E. Shafranjuk, "Directional photoelectric current across the bilayer graphene junction," Journal of Physics: Condensed Matter, vol. 21, no. 1, Article ID 015301, 2009.

[38] V. Ryzhii, V. Mitin, M. Ryzhii, N. Ryabova, and T. Otsuji, "Device model for graphene nanoribbon phototransistor," Applied Physics Express, vol. 1, no. 6, Article ID 063002, 3 pages, 2008. 

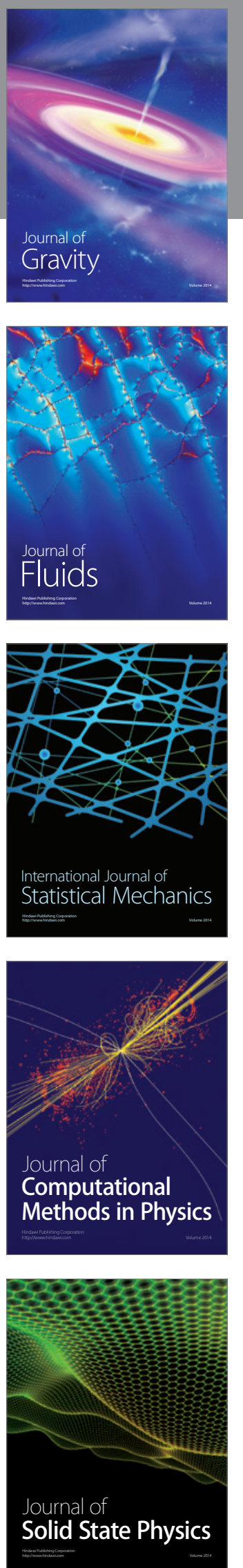

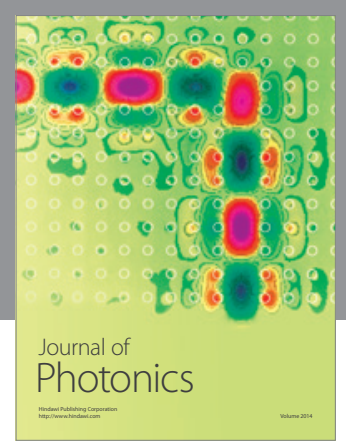

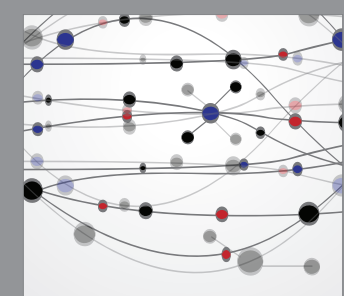

The Scientific World Journal
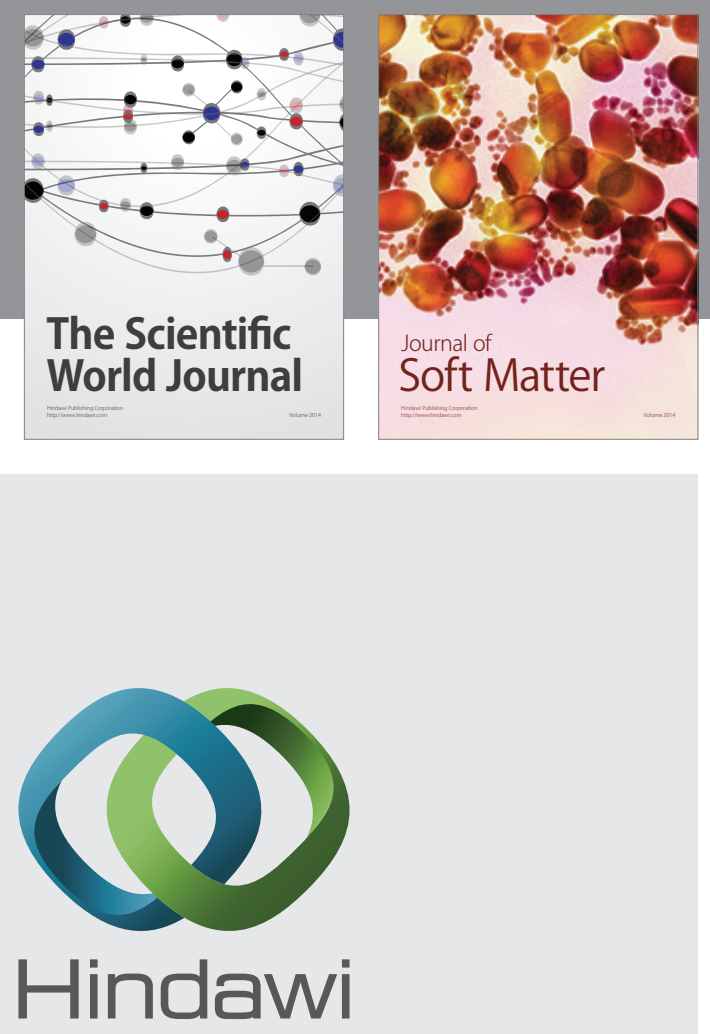

Submit your manuscripts at

http://www.hindawi.com
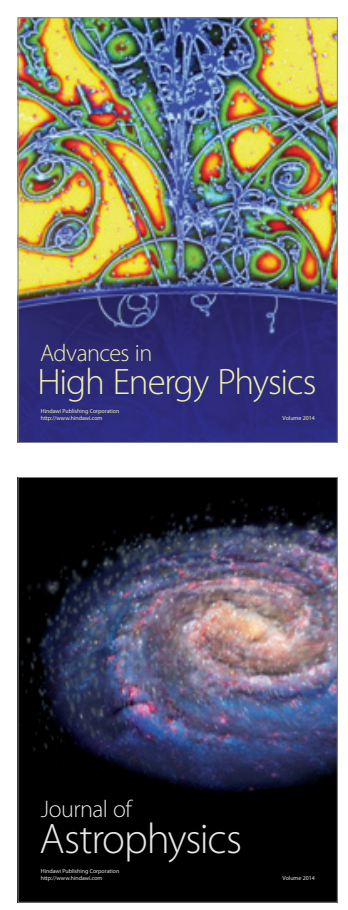
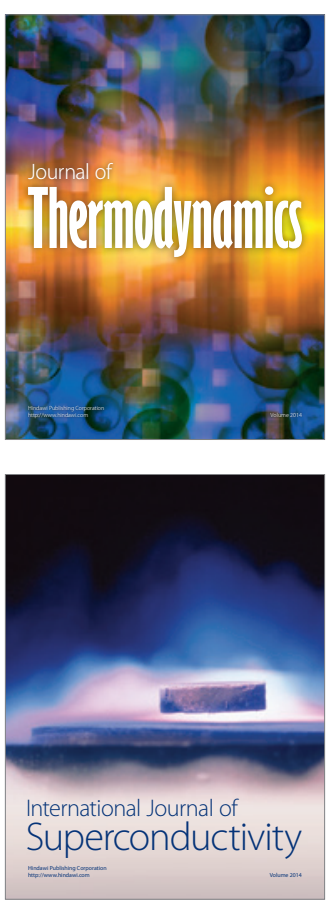
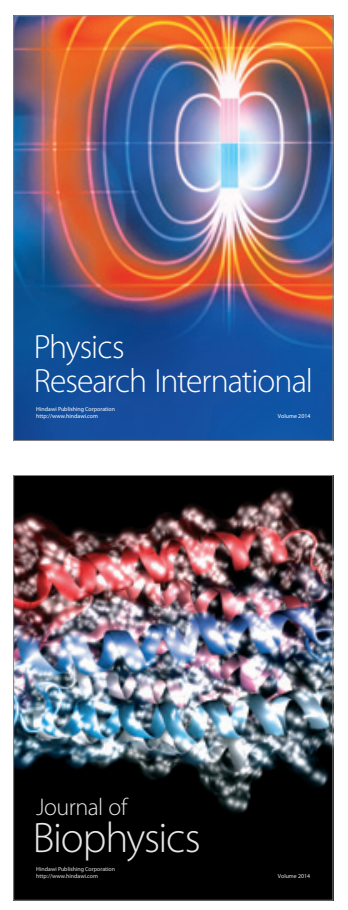
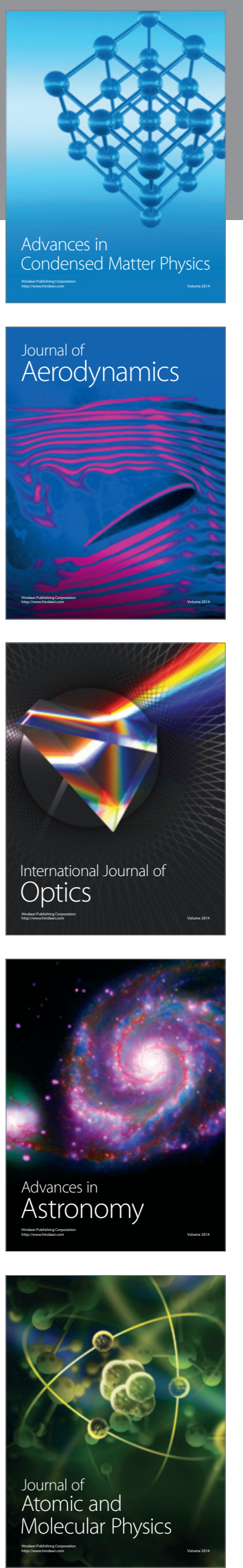